\title{
Presenilin 1 intronic polymorphism is not associated with Alzheimer type neuropathological changes or sporadic Alzheimer's disease
}

\author{
N Sodeyama, Y Itoh, N Suematsu, M Matsushita, E Otomo, H Mizusawa, M Yamada
}

\begin{abstract}
Background-A genetic association between the presenilin 1 (PS-1) intronic polymorphism and sporadic Alzheimer's disease has been a matter of controversy. Recent findings have suggested that the PS-1 polymorphism is not associated with Alzheimer's disease or amyloid $\beta$-protein (Aß) deposition in brains from patients with Alzheimer's disease.

Objectives-To elucidate the influence of the PS-1 polymorphism on Alzheimer type neuropathological changes and the development of Alzheimer's disease, the relation between the PS-1 polymorphism and quantitative severity of Alzheimer type neuropathological changes in the brains from patients with Alzheimer's disease and non-demented subjects was studied.
\end{abstract}

Methods-The PS-1 and apolipoprotein E (ApoE) genotypes, were examined, together with the densities of the senile plaques, senile plaques with dystrophic neurites, and neurofibrillary tangles in the brains from 36 postmortem confirmed patients with sporadic Alzheimer's disease and 86 non-demented subjects. Association of the PS-1 polymorphism with sporadic Alzheimer's disease and ages at onset and duration of illness in Alzheimer's disease was also examined.

Results-The PS-1 polymorphism was not associated with the senile plaques, senile plaques with dystrophic neurites, or neurofibrillary tangles in Alzheimer's disease or non-demented subjects. There was no association of the PS-1 intronic polymorphism with Alzheimer's disease, ages at onset, or durations of illness in Alzheimer's disease. The results remained nonsignificant even when the PS-1 genotype groups were divided into the subgroups withdifferent ApoE $\varepsilon 4$ status.

Conclusions-The PS-1 intronic polymorphism does not itself have a direct causal role in the formation of Alzheimer type neuropathological changes or in the development of sporadic Alzheimer's disease. (f Neurol Neurosurg Psychiatry 1998;64:548-551)
Keywords: Alzheimer's disease; presenilin 1; neuropathological change

The presenilin 1 (PS-1) gene accounts for about $25 \%$ of early onset familial Alzheimer's disease. ${ }^{1}$ Wragg et al reported a genetic association between the PS-1 intronic polymorphism and sporadic Alzheimer's disease. ${ }^{2}$ This relation has, however, been a matter of controversy. ${ }^{3-8}$ The general topology of the putative PS-1 protein suggests that it is an integral membrane protein, such as a receptor, a channel protein, or a structural membrane protein. ${ }^{1}$ Although the functions of the PS-1 protein have not been defined, the accumulated PS-1 protein was found in the senile plaques in the brains of patients with Alzheimer's disease, suggesting that the PS-1 protein may have an effect on the formation of the senile plaques. $^{9}$ Lemere et al disclosed increased deposition of amyloid $\beta$-protein $(A \beta)$ ending at residue $42(\mathrm{~A} \beta 42)$ in the brains from patients with familial Alzheimer's disease with the PS-1 mutation compared with those from sporadic patients with Alzheimer's disease. ${ }^{10}$ Borchelt et al showed an increased $\mathrm{A} \beta 42 / \mathrm{A} \beta 40$ ratio in the conditioned media of cell lines expressing familial Alzheimer's disease linked PS-1 variants and in the brain of the transgenic mouse expressing familial Alzheimer's disease linked PS-1 variants. ${ }^{11}$ These studies support the hypothesis that the mechanism by which familial Alzheimer's disease linked PS-1 mutations cause Alzheimer's disease is to increase A $\beta 42$ concentrations in brain tissue, resulting in the formation of senile plaques. Murphy et al showed that the antibody to the PS-1 protein reacted with the neurofibrillary tangles. ${ }^{12}$ Bouras et al reported that the PS-1 expression was decreased in neurons bearing the neurofibrillary tangles, suggesting that absence of the PS-1 protein was associated with increased neuronal vulnerability to the degenerative process. ${ }^{13}$ These findings suggest a possible correlation between the PS-1 genotype and severity of Alzheimer type neuropathological changes.

Recently, Mann et al reported that the PS-1 intronic polymorphism did not influence the amount or molecular form of $A \beta$ in the brains 
Table 1 PS-1 Genotypes and the densities of senile plaques (SPs), senile plaques with dystrophic neurites (NPs), and neurofibrillary tangles (NFTs) in the hippocampus and superior temporal gyrus in patients with Alzheimers disease (AD) and non-demented subjects, and ages at onset and durations of illness in $A D$

\begin{tabular}{|c|c|c|c|c|c|c|c|c|}
\hline \multirow[b]{2}{*}{ Presenilin 1 genotype } & \multicolumn{4}{|l|}{$A D(n=36)$} & \multicolumn{4}{|c|}{ Non-demented $(n=86)$} \\
\hline & $1 / 1(n=13)$ & $1 / 2(n=18)$ & $2 / 2(n=5)$ & $p$ Values & $1 / 1(n=33)$ & $1 / 2(n=41)$ & $2 / 2(n=12)$ & $p$ Values \\
\hline \multicolumn{9}{|l|}{ Hippocampus: } \\
\hline SPs & $16.0(7.5,21.5)$ & $20.8(12.4,23.0)$ & $12.3(11.8,28.5)$ & 0.48 & $0.0(0.0,0.0)$ & $0.0(0.0,1.0)$ & $0.0(0.0,3.2)$ & 0.73 \\
\hline NPs & $11.2(7.2,16.0)$ & $18.0(11.0,21.8)$ & $12.0(10.5,28.5)$ & 0.29 & $0.0(0.0,0.0)$ & $0.0(0.0,0.7)$ & $0.0(0.0,0.7)$ & 0.86 \\
\hline NFTs & $38.0(11.9,64.7)$ & $53.6(35.4,62.4)$ & $52.0(45.8,66.3)$ & 0.22 & $0.4(0.2,4.1)$ & $0.8(0.2,5.4)$ & $1.0(0.2,2.9)$ & 0.91 \\
\hline \multicolumn{9}{|c|}{ Superior temporal gyrus: } \\
\hline SPs & $73.0(30.1,106.3)$ & $72.7(44.8,100.0)$ & $58.8(52.3,83.0)$ & 0.92 & $0.0(0.0,3.8)$ & $0.0(0.0,8.3)$ & $0.1(0.0,7.0)$ & 0.29 \\
\hline NPs & $14.0(7.9,22.0)$ & $12.4(7.0,27.0)$ & $21.3(14.8,43.1)$ & 0.45 & $0.0(0.0,1.8)$ & $0.0(0.0,1.9)$ & $0.0(0.0,2.2)$ & 0.80 \\
\hline NFTs & $4.2(0.8,12.1)$ & $2.2(0.4,12.2)$ & $6.5(5.2,11.3)$ & 0.37 & $0.0(0.0,0.0)$ & $0.0(0.0,0.0)$ & $0.0(0.0,0.0)$ & 0.94 \\
\hline Age at onset & $82.0(77.8,84.5)$ & $76.5(73.0,84.0)$ & $78.0(71.3,85.8)$ & 0.62 & & & & \\
\hline Duration of illness & $5.0(2.0,8.5)$ & $5.5(4.0,10.0)$ & $4.0(2.8,8.5)$ & 0.74 & & & & \\
\hline
\end{tabular}

Values are medians $\left(25,75\right.$ percentiles). The density represents the average counts in $2.56 \mathrm{~mm}^{2}$ for the SPs and NPs and in 0.64 mm for the NFTs.

from patients with Alzheimer's disease. ${ }^{14}$ To elucidate whether and how the PS-1 polymorphism influences the development of Alzheimer's disease and Alzheimer type neuropathological changes, it is important to know the relation between the PS-1 polymorphism and Alzheimer type neuropathological changes in detail in the brains from patients with Alzheimer's disease and non-demented subjects in a single ethnic population. If this polymorphism could have a role in the onset of Alzheimer's disease, the formation of Alzheimer type neuropathological changes found in the brains from either patients with Alzheimer's disease or non-demented subjects may be accelerated under the influence of the polymorphism. In this study, we have examined the PS- 1 intronic polymorphism and severity of the senile plaques, senile plaques with dystrophic neurites, and neurofibrillary tangles in postmortem confirmed patients with sporadic Alzheimer's disease and non-demented subjects from a necropsy series of elderly Japanese people.

\section{Subjects and methods}

SUBJECTS

The subjects were 36 postmortem confirmed patients with sporadic Alzheimer's disease (age at death, 62-95 years; median (25 percentile, 75 percentile), $87.5(80.0,89.0)$ years) and 86 non-demented subjects (age at death, 65 to 101 years; median (25 percentile, 75 percentile), 85.5 (79.0, 92.0) years) from a necropsy series at Yokufukai Geriatric Hospital, Tokyo. All subjects were Japanese. Ages at death were not significantly different between the two groups ( $\mathrm{p}=0.827$; Mann-Whitney $U$ test). Non-demented subjects were without any neurodegenerative disorders. Patients with Alzheimer's disease satisfied the neuropathological criteria of the Consortium to Establish a Registry for Alzheimer's disease. ${ }^{15}$ Clinical diagnosis of Alzheimer's disease at onset was based on the criteria of the NINCDS-ADRDA and DSM III-R. ${ }^{16}{ }^{17}$

NEUROPATHOLOGICAL EXAMINATION

The densities of the senile plaques, senile plaques with dystrophic neurites, and neurofibrillary tangles were quantified using formalin fixed, paraffin embedded sections stained with methenamine Bodian from the hippocampus and superior temporal gyrus of the brains from all subjects. The methenamine Bodian stain could detect all types of senile plaques including the diffuse plaques with almost equal sensitivity to the $A \beta$ immunohistochemistry, which we had confirmed in a previous study. ${ }^{18}$ For diffuse plaques, those smaller than $25 \mu \mathrm{m}$ in diameter were excluded from the count. Ten $\times 100$ microscopical fields (field size 2.56 $\mathrm{mm}^{2}$ ) were randomly chosen and counted for the senile plaques and senile plaques with dystrophic neurites, and ten $\times 200$ microscopical fields (field size $0.64 \mathrm{~mm}^{2}$ ) for the neurofibrillary tangles. The results were then averaged. The quantification was performed without information about the PS-1 and apolipoprotein E (ApoE) genotypes.

\section{IDENTIFICATION OF POLYMORPHISMS}

Genomic DNA was extracted from the frozen brain tissue of all subjects by digestion in lysis buffer with RNase and proteinase $\mathrm{K}$, followed by phenol/chloroform extraction. For the PS-1 intronic polymorphism, genomic DNA was amplified by polymerase chain reaction (PCR) with oligonucleotide primers described by Wragg et al, and then were digested with Bam $\mathrm{HI}^{2}{ }^{2}$ The resulting DNA fragments were resolved on a $3 \%$ agarose gel. For the ApoE polymorphism, the PCR was performed as described by Hixson et al. ${ }^{19}$ The PCR product was digested with Hha $I$ and electrophoresed Bam on an $8 \%$ polyacrylamide gel. The results were visualised by ethidium bromide staining.

\section{STATISTICAL ANALYSIS}

The densities of the senile plaques, senile plaques with dystrophic neurites, and neurofibrillary tangles in each region for both groups, and ages at onset and durations of illness for Alzheimer's disease were compared among the PS-1 genotypes with a Kruskal-Wallis test as the data were not normally distributed. The Mann-Whitney $U$ test was used when the PS-1 $1 / 2$ and $2 / 2$ genotypes were combined into a single category. The genotypic and allelic distribution of the PS-1 intronic polymorphism in total cases were analysed with a $\chi^{2}$ test. Genetic associations between the $\varepsilon 4$ allele and Alzheimer's disease in all cases or in the subgroups divided into the PS-1 genotypes were analysed by a $\chi^{2}$ test. In addition, similar statistical analyses were done among the PS-1 genotype or allele subgroups of ApoE $\varepsilon 4$ allele status. Significance was defined as two tailed probabilities of $<0.05$. All analyses were 
Table 2 Genotypic and allelic distribution of the PS-1 intronic polymorphism and the ApoE $\varepsilon 4$ status

\begin{tabular}{|c|c|c|c|c|c|c|c|c|c|}
\hline & \multicolumn{5}{|c|}{ Presenilin 1 genotype } & \multicolumn{4}{|c|}{ Presenilin 1 allele } \\
\hline & $1 / 1$ & $1 / 2$ & $2 / 2$ & $\chi^{2}$ & $p$ Value & 1 & 2 & $\chi^{2}$ & p Value \\
\hline $\mathrm{AD}(\mathrm{n}=36)$ & 13 & 18 & 5 & 0.06 & 0.97 & 44 & 28 & 0.03 & 0.87 \\
\hline $\begin{array}{l}\text { Non-demented }(\mathrm{n}=86) \\
\varepsilon 4(-):\end{array}$ & 33 & 41 & 12 & & & 107 & 65 & & \\
\hline $\mathrm{AD}(\mathrm{n}=21)$ & 6 & 10 & 5 & 1.59 & 0.45 & 22 & 20 & 1.55 & 0.21 \\
\hline $\begin{array}{l}\text { Non-demented }(\mathrm{n}=73) \\
\varepsilon 4(+):\end{array}$ & 29 & 34 & 10 & & & 92 & 54 & & \\
\hline $\mathrm{AD}(\mathrm{n}=15)$ & 7 & 8 & 0 & 2.76 & 0.25 & 22 & 8 & 1.52 & 0.22 \\
\hline Non-demented $(n=13)$ & 4 & 7 & 2 & & & 15 & 11 & & \\
\hline
\end{tabular}

performed using computer software (StatView J-4.5, Abacus Concepts, Berkeley, CA, USA).

\section{Results}

Table 1 shows the PS-1 genotypes and the densities of the senile plaques, senile plaques with dystrophic neurites, and neurofibrillary tangles in the hippocampus and superior temporal gyrus in patients with Alzheimer's disease or non-demented subjects, and ages at onset and durations of illness for Alzheimer's disease. There were no genotypic associations of the PS-1 genotypes with the densities of the senile plaques, senile plaques with dystrophic neurites, or neurofibrillary tangles in either of the regions in either group. The PS-1 genotypes did not also influence ages at onset, or duration of illness in Alzheimer's disease. In addition, when the PS-1 genotype groups were divided into the subgroups of ApoE $\varepsilon 4$ status, the PS-1 genotypes did not influence the densities of the senile plaques, senile plaques with dystrophic neurites, or neurofibrillary tangles in either of the regions in either group, ages at onset, or durations of illness in Alzheimer's disease (data not shown). Genetic association of the PS-1 genotypes with Alzheimer type neuropathological changes, ages at onset, or durations of illness in Alzheimer's disease remained nonsignificant even when we combined the PS-1 $1 / 2$ and $2 / 2$ genotypes into a single category (data not shown).

Table 2 shows the genotypic and allelic distribution of the PS-1 intronic polymorphism, and the ApoE \&4 status. There were no significant differences in the frequencies of the PS-1 genotypes or alleles between the two groups. Even when the PS-1 genotype or allele groups were divided into subgroups of ApoE $\varepsilon 4$ allele status, there were no significant differences in the frequencies of the PS-1 genotypes or alleles between the Alzheimer's disease group and the non-demented group. Genetic association of the PS-1 genotypes with sporadic Alzheimer's disease remained nonsignificant even when we combined the PS-1 $1 / 2$ and $2 / 2$ genotypes into a single category (data not shown). There was no significant association between the PS-1 and ApoE genotypes in Alzheimer's disease (data not shown).

The presence of the $\varepsilon 4$ allele was associated with sporadic Alzheimer's disease in total cases $(p=0.002)$, and in patients with either the PS-1 genotype $1 / 1$ or $1 / 2$, but not $2 / 2(1 / 1, p=0.003$; $1 / 2, p=0.03 ; 2 / 2, p=0.33)$. The PS-1 genotype frequencies in our control subjects were not significantly different from those in the other previous reports. ${ }^{2-68}$ This result, with fewer samples than were included in these data was reported previously. ${ }^{20}$

\section{Discussion}

Our results disclosed lack of association of the PS-1 intronic polymorphism with Alzheimer type neuropathological changes in patients with Alzheimer's disease or non-demented subjects. Recently, Mann et al reported that the PS-1 intronic polymorphism did not influence the amount of $A \beta$ in Alzheimer's disease. ${ }^{14}$ Our results support this finding. Further, we showed that the PS-1 polymorphism was not associated with the other neuropathological hallmarks of Alzheimer's disease, the senile plaques with dystrophic neurites or neurofibrillary tangles, in the Alzheimer's disease group or the non-demented group. These data indicate that the PS-1 polymorphism does not influence the formation of the Alzheimer type neuropathological changes which are linked to the development and progression of Alzheimer's disease.

In our study, the PS-1 intronic polymorphism was also not associated with the development of sporadic Alzheimer's disease, ages at onset, or duration of illness in Alzheimer's disease. Although our sample size was small, the PS-1 genotype frequencies in our control subjects were not significantly different from those in the other reports, ${ }^{2-68}$ suggesting that our sample population was not biased. Scott et al and Pérez-Tur et al found no definite differences between Alzheimer's disease and control groups either in genotypic or allelic distribution. $^{67}$ Wragg et al also found no increased risk of Alzheimer's disease for genotype 1/1 in African Americans. ${ }^{2}$ Tysoe et al showed lack of association of the PS-1 polymorphism with postmortem confirmed Alzheimer's disease. ${ }^{8}$ The association between the PS-1 intronic polymorphism and sporadic Alzheimer's disease in some populations may be recognised as linkage disequilibrium with relevant variability in the PS-1 or other adjacent gene. ${ }^{2-5}$

The ApoE $\varepsilon 4$ allele was associated with sporadic Alzheimer's disease in patients with either the PS- 1 genotype $1 / 1$ or $1 / 2$ in our study. Lack of association in patients with the PS-1 genotype $2 / 2$ was probably due to the small sample size. These findings indicate that the PS-1 intronic polymorphism does not affect association between the $\varepsilon 4$ allele and sporadic Alzheimer's disease. 
In conclusion, our data suggest that the PS-1 intronic polymorphism does not itself have a direct causal role in the formation of Alzheimer type neuropathological changes in the brain or in the development of sporadic Alzheimer's disease. The PS-1 1/1 genotype or 1 allele is not a critical factor in accelerating onset of sporadic Alzheimer's disease. Our results should be verified in larger necropsy series of patients with Alzheimer's disease and nondemented subjects, and in other ethnic populations.

This work was supported in part by Health Science Research Grants (Mechanism of Abnormal Deposition in Dementia Brain) of the Ministry of Health and Welfare of Japan to MY.

1 Sherrington R, Rogaev EI, Liang Y, et al. Cloning of a gene bearing missense mutations in early-onset familial Alzheimer's disease. Nature 1995;375:754-60.

2 Wragg $M$, Hutton $M$, Talbot $C$, the Alzheimer's disease Collaborative Group. Genetic association between intronic polymorphism in presenilin-1 gene and late-onset Alzheimpolymorphism in presenilin-1 gene and
er's disease. Lancet 1996;347:509-12.

3 Higuchi S, Muramatsu T, Matsushita S, et al. Presenilin-1 polymorphism and Alzheimer's disease. Lancet 1996;347: polym 1186 .

4 Kehoe P, Williams J, Holmans P, et al. Association between a PS-1 intronic polymorphism and late onset Alzheimer's disease. Neuroreport 1996;7:2155-8.

5 Kehoe P, Williams J, Lovestone S, et al, and the UK Alzheimer's Disease Collaborative Group. Presenilin-1 polymorphism and Alzheimer's disease. Lancet 1996;347:1185.

6 Pérez-Tur J, Wavrant-De Vrieze F, Lambert JC, et al, and the Alzheimer's Study Group. Presenilin-1 polymorphism and Alzheimer's disease. Lancet 1996;347:1560-1.

7 Scott WK, Growdon JH, Roses AD, et al. Presenilin-1 polymorphism and Alzheimer's disease. Lancet 1996;347:1186-
8 Tysoe C, Whittaker J, Cairns NJ, et al. Presenilin-1 intron 8 polymorphism is not associated with autopsy-confirmed late-onset Alzheimer's disease. Neurosci Lett 1997;222:68-9.

9 Wisniewski T, Palha JA, Ghiso J, et al. S182 protein in Alzheimer's disease neuritic plaques. Lancet 1995;346:1366.

10 Lemere CA, Lopera F, Kosik KS, et al. The E280A presenilin 1 Alzheimer mutation produces increased $\mathrm{A} \beta 42$ deposition and severe cerebellar pathology. Nat Med 1996;2: 1146-50.

11 Borchelt DR, Thinakaran G, Eckman CB. Familial Alzheimer's disease-linked presenilin 1 variants elevate A $\beta 1-42 / 1-40$ ratio in vitro and in vivo. Neuron 1996;17: A $\beta 1-42 / 1$

12 Murphy GM, Forno LS, Ellis WG, et al. Antibodies to presenilin proteins detect neurofibrillary tangles in Alzheimer's disease. Am F Pathol 1996;149:1839-46.

13 Bouras C, Giannakopoulos P, Schioi J, et al. Presenilin-1 polymorphism and Alzheimer's disease. Lancet 1996;347: 1185-6.

14 Mann DMA, Pickering-Brown SM, Bayatti NN, et al. An intronic polymorphism in the presenilin-1 gene does not influence the amount or molecular form of the amyloid $b$ infuence the amount or molecular form of the amyloid b protein deposite

15 Mirra SS, Heyman A, McKeel D, et al. The consortium to establish a registry for Alzheimer's disease (CERAD). Part II. Standardization of the neuropathologic assessment of Alzheimer's disease. Neurology 1991;41:479-86.

16 American Psychiatric Association, Committee on Nomenclature. In: Diagnostic and statistical manual of mental disorders, 3rd ed. Washington, DC: APA, 1987.

17 McKhann G, Drachman D, Folstein M, et al. Clinical diagnosis of Alzheimer's disease: report of the NINCDSADRDA Work Group under the auspices of Department of Health and Human Services Task Force on Alzheimer's Disease. Neurology 1984;34:939-44.

18 Yamada M, Itoh Y, Otomo E, et al. Dementia of the Alzheimer type and related dementias in the aged: DAT subgroups and senile dementia of the neurofibrillary tangle type. Neuropathology 1996;16:89-98.

19 Hixson JE, Vernier DT. Restriction isotyping of human apolipoprotein $\mathrm{E}$ by gene amplification and cleavage with Hha I. F Lipid Res 1990;31:545-8.

20 Itoh Y, Yamada M. Apolipoprotein E and the neuropathology of dementia. N Engl F Med 1996;334:599-600. 